\title{
Exploring perceptions and attitudes of Black sub-Sahara African (BSSA) migrants towards residential care in England.
}

\begin{tabular}{|r|l|}
\hline Journal: & International Journal of Migration, Health and Social Care \\
\hline Manuscript ID & IJMHSC-12-2019-0097.R1 \\
\hline Manuscript Type: & Academic Paper \\
\hline Keywords: & Residential Care, sub-Sahara, African, Migrants, culture \\
\hline \multicolumn{2}{|l}{} \\
\end{tabular}




\section{Acknowledgements}

Our special thanks to all individuals from the black Sub-Sahara African communities who took part and shared their perceptions and attitudes towards residential care in this research and all community groups that facilitated this study. This study received no funding and all authors declare no conflict of interest. 
Table 1: Demographic Profile of Research Participants

\begin{tabular}{|l|l|l|l|l|l|}
\hline $\mathbf{s} / \mathbf{n}$ & Countries & Aged range & Females & Males & $\begin{array}{l}\text { Total no. of participants } \\
\text { from each country }\end{array}$ \\
\hline 1. & Angola & $67-83$ years & 5 & 2 & 7 \\
\hline 2. & Benin & $65-91$ years & 1 & 2 & 3 \\
\hline 3. & Burkina Faso & $68-85$ years & 2 & 5 & 7 \\
\hline 4. & Burundi & $65-81$ years & - & 1 & 1 \\
\hline 5. & Cameroon & $73-94$ years & 4 & 2 & 6 \\
\hline 6. & Cape Verde & $66-89$ years & 1 & 5 & 6 \\
\hline 7. & Chad & $65-69$ years & - & 2 & 2 \\
\hline 8. & Congo & $65-74$ years & 3 & 5 & 8 \\
\hline 9. & Congo (Democratic Republic) & $68-82$ years & 1 & 4 & 5 \\
\hline 10. & Cote d'lvoir & $65-77$ years & 2 & 2 & 4 \\
\hline 11. & Equatorial Guinea & $78-85$ years & 1 & - & 1 \\
\hline 12. & The Gambia & $65-95$ years & 5 & 7 & 12 \\
\hline 13. & Ghana & $71-93$ years & 7 & 3 & 10 \\
\hline 14. & Guinea & $76-83$ years & 2 & 4 & 6 \\
\hline 15. & Kenya & $65-88$ years & 8 & 1 & 9 \\
\hline 16. & Malawi & $65-84$ years & 7 & 2 & 9 \\
\hline 17. & Mali & $67-96$ years & 3 & 5 & 8 \\
\hline 18. & Niger & $80-87$ years & - & 2 & 2 \\
\hline 19. & Nigeria & $65-89$ years & 16 & 11 & 27 \\
\hline 20. & Zimbabwe & $66-72$ years & 4 & 7 & 11 \\
\hline
\end{tabular}




\section{Background}

Care support for the elderly is a broad reaching concept, not all support for the elderly is in form of residential care. Sometimes the care may exclude statutory health and housing service. The support for the elderly can be categorised into emotional support, informational support and instrumental support. Statistics collated in 2011 suggest that more than 12 million people in the UK are aged 65 and above (Office of National Statistics (ONS) 2011). Since the start of the $19^{\text {th }}$ century, the UK has seen mortality rates decline and life expectancy increase (Office of National Statistics (ONS) 2018). With more young people and their families becoming more pre-occupied with work and professional fulfilment, there are very few people committing to taking care of the elderly at home (Centre for Ageing Better, 2017). This has increased the number of elderly people being put into residential care. The run up to the new millennium witnessed a change in British population demography with the arrival of many Africans from the Black Sub-Sahara African (BSSA) countries owing to economic meltdown and internal strife (ONS, 2014). Sub-Sahara Africa is made up of all African countries that are located south of the Sahara Desert. As of 2018, eight percent of people aged 60 and above in England and Wales were BME compared to 14\% of the total population (ONS 2018). Based on the above assertions, this has implications for residential care for BSSA elderly people.

\section{Although BSSA communities have participated in many community inclusion} initiatives, many studies among hard to reach migrants have reported that, having other priorities such as extended family responsibilities including caring for grandchildren (normally among women), schedule or timetabling conflicts or lack of time prevented participation and engaging with wider health and social care services (Dyall et al., 2013) . Some elderly people from ethnic minority groups may be reluctant to receive home visits as part of preserving their privacy (Holland et al., 2008). There is also further evidence of low uptake in health promotion among this group making it difficult for health professionals to engage them (Martinez et al., 2009). In some occasions, participants perceived that the health assessments were too long to attend and sometimes asked for a partial assessment making it difficult to make decisions, which are beneficial to their health and well-being (Dyall et al., 2013). Cultural and language barriers may also work against the desire for migrants to use services, which are deemed unfriendly in their own community (National Health Service (NHS), 2014). Lack of confidence and fear of embarrassment during personal care often by a staff member of the opposite sex may sometimes reduce uptake of residential care services 
from individuals whose cultural values are sensitive (Maybin, Charles and Honeyman, 2016).

Access to residential health and social care for all vulnerable groups from a diverse background is key to achieving positive health outcomes. Currently there are concerns that some vulnerable hard to reach groups in the population have poorer access to health and social care than others (The Centre for Mental Health, 2002). A range of factors influence access to residential health and social care for hard to reach groups, which are sometimes disadvantaged when they seek services because they may present late, have difficulty in getting their issues across, or have problems such as other chronic conditions that makes it difficult for them to access these services (Department of Health, 2009). Sometimes there is not enough capacity in the system to meet everyone's needs especially new emerging communities like the BSSA communities, which became more visible in the run up to the new millennium (Office of National Statistics 2014). Currently, the BSSA communities who became visible in the UK more than fifteen years ago are slowly aging and there are strong indications that they may soon need some health and social care support in the form of residential care. This paper is therefore going to explore perceptions and attitudes towards residential care provision among BSSA aged sixty-five years and beyond.

\section{Methodology}

The Silences Framework (Serrant-Green, 2011) guided this research. The Framework asserts that reality is not objective or fixed but rather human beings scripts the social world in communities at a particular time (Serrant-Green, 2011). The Framework put an emphasis on the 'Screaming Silences' in individual and group interpretations of experiences that can be qualified as 'truth'. Screaming Silences can be viewed as a marginalised discourse that fills identified research gaps (Ifekwunigwe, 1997). In this paper, silences were explored in relation to perceptions and attitudes of BSSA communities towards residential care for the elderly in the West Midlands. The Screaming Silences in relation to cultural practices and beliefs within BSSA communities were explored to ascertain perceptions and attitudes towards residential care for the elderly.

An explorative qualitative approach was utilised for this study using focus group discussions and one- to-one follow up semi-structured interviews. Data was collected from BSSA communities living in the cities of Birmingham, Wolverhampton and Coventry in the West Midlands region. These areas have high percentages of BSSA communities. The study participants were selected through purposeful sampling based on location, sex, age and origin 
to include BSSA men and women aged 65 and over (Patton, 2002).

Eighteen focus group discussions were conducted, six in each of the three cities with each focus group made up of eight people, with an equal sex balance. $(\mathbf{N}=\mathbf{1 4 4})$. Each of the focus groups was followed up with two one-to-one semi-structured follow up interviews. $(\mathbf{N}=\mathbf{3 6})$ A small number of participants declined to take part mainly because of discomfort with the subject, however, their places were taken up by others from the same communities. Ethical approval was granted by The Heart of England Foundation Trust and the University of Wolverhampton research ethics committee.

The research participants were identified and contacted through the BSSA community-based groups and the local health organisations in the cities of Birmingham, Wolverhampton and Coventry. Participation in the research study was voluntary and all research participants gave a written consent. Table 1 below shows participants profile by origin age and sex.

\section{Table 1: Demographic Profile of Research Participants}

The research participants discussed cultural issues concerning perceptions and attitudes towards residential care among BSSA communities. They appraised and commented on current social service residential care for the elderly programmes suggesting opportunities for further development. The roles of families and cultural values in residential care were scrutinised and suggestions for inclusivity were discussed.

The focus group discussions with the research participants lasted for one hour while the oneto-one semi structured follow up interviews lasted for 30-45 minutes. All focus group discussions and one-to-one follow up interviews were audio recorded and transcribed verbatim. The transcribed data was subjected to the four phases of the Silences Framework shown below (Serrant-Green, 2011).

- Phase 1 - After transcription, the outputs from the interviews and focus group discussions were analysed by the researcher and recurrent themes were identified as the preliminary findings from the study.

- Phase 2 - The preliminary findings from phase 1 were reviewed by the research participants. Reflections on the early findings from the participants were used to enhance further critique, confirming or refuting the findings from phase 1. A discussion of the silences (Findings) was formulated. 
- Phase 3 - A further analysis of the findings from phase 2 was undertaken in this stage by social networks of research participants. The participants in this phase were drawn from the BSSA communities that had not taken part in the focus group discussions and one to one follow up interviews. The aim was to consolidate findings from phase 2 with a critical indirect associative eye.

- Phase 4 - Finally the researcher reflected on the findings from phase 3, revisiting, reviewing and developing emerging themes which formed the final output of this study.

\section{Results}

Following the organisation and analysis of the data exploring perceptions and attitudes towards residential care, the following themes supported by narrative extracts emerged:

\section{Confinement}

Most of the research participants viewed residential care as being confined in a very limited environment in terms of movement and proximity to friends. Most of the BSSA elderly people are used to spending most of their time walking about talking to neighbours and friends. However, the residential care with normally a small room within a big house does not provide for such an environment and freedom to move about.

"Honestly I feel that residential care is about being confined in a very restricted environment among people you don't even know, I can't spent the whole day in a room, I need friends to talk to and move around for fresh air" A 70-year old man from Zimbabwe.

"I grew up talking to neighbours and visiting friends living nearby, I can't live in a place where I will be held for days without going out to see and talk to the people I grew up with. A 75-year-old woman from Kenya.

\section{Lack of ownership}

The research participants felt that residential care takes away a sense of ownership and legacy in the immediate environment. There was a feeling that it separated them with all the things that they had worked to achieve like property and children. They reported having a sense that the place belonged to someone taking charge of their lives.

"I feel that this residential care reduces me to someone who has nothing in life, that a small room cannot contain all I have achieved in life. I definitely need to show what I have 
achieved to all those who visit me" A 69-year old woman from Zambia.

"I can't just live a home that I have built to live in a very small room owned by someone; honestly people will start to question whether I have achieved anything during my working time" A 72-year old man from Mozambique.

\section{Culture and food}

The research participants reported that residential care does not provide food that they have grown up eating back home in Africa. They also felt that there was a big misunderstanding with regards to different foods black people eat and that there is a tendency to assume that all black people eat rice and peas as their staple diet.

"When I visited my friend at one of the residential homes, the nurses told me that they had cooked rice and peas for my friend as a special traditional dish, honestly my friend is not from the Caribbean he would do well with sadza (a maize meal dish) and chicken" A 66-year old woman from Zimbabwe.

"I grew up eating hot food every time, honestly I can't eat sandwiches for dinner and go to bed. I will be hungry the whole night" A 68-year old man from Uganda.

\section{Culture and personal care}

Research participants felt that it was not within their culture to be personally cared for by a person not related to them. They felt embarrassed and neglected to receive care from a person not related to them, which is what normally happens.

"I can't be assisted with personal care by a person I don't know. In my culture, I am supposed to be helped by my personal relative. What will the people think about me when they see me stuck in a care home? It will really be embarrassing" A 67-year old woman from the Democratic Republic of Congo.

"It's embarrassing to be cared for by people who are not related to me. What will people say about me and my relatives? I would rather stay alone than to be in a care home where no one knows me” A 68-year old man from Rwanda.

\section{Death and dying}

The research participants felt that when they are old and nearing death, it should happen around people they are related to. They also felt that their palliative care should be performed 
by their close relatives in line with their cultural beliefs.

"I should die around my relatives as opposed to strangers. Only my relatives should care for me in my last days and once you go into residential care all this will never happen" A 72year old man from Angola.

"My culture does not allow me to be cared for by strangers in my last days, it will definitely be an embarrassment to the community when I die in the care of strangers, honestly residential care is not for me" A 71-year old woman from South Africa.

\section{Sign of neglect}

The research participants also equated living in residential care as a sign of being rejected by your own relatives. They felt that residential care among BSSA communities was depicted with the stigma of being rejected associated with poverty and poor cohesion within the family.

"I can't live in residential care as though I have no relatives, that's a place of irresponsible people with broken down relations and I am not one of them" A 68-year old man from Swaziland.

"Only those people who were not responsible and well behaved will seek residential care manned by strangers, honestly I was responsible and send my children to school. Now it is their turn to look after me. I can't afford to be a laughing stock" A 65-year old woman from Zambia.

\section{Poor inclusivity in activities and loneliness}

The research participants felt that residential care homes did not have inclusive activities to take into account the needs of black people. They felt that most of the activities are based on the mainstream society with little or no diversity at all.

"Honestly I can't go to a place where I struggle to cope with activities because they are new to me. None of the games or music played is familiar, only those born and bred in this country will enjoy the activities. I would rather live alone than to go in residential care” $\mathbf{A}$ 74-year old man from Lesotho.

"My friend in residential care told me that she is viewed as dafty because she can't answer any quiz questions or sing along any song played by the activity lady, honestly how can she answer questions about Britain and Europe, when she spent most of her time in Botswana 


\section{and Africa” A 67-year old woman from Namibia.}

\section{Discussion}

In the African context, elderly people are regarded as the custodians of the community and revered by all (Torres, 2012). This places a special treatment on the elderly with the freedom to walk about and talk to neighbours as part of spending their day. If they want to go anywhere, a young person normally, a relative acting as a guide can accompany them. It is against this background that the research participants viewed living in residential care as confinement, owing to the restricted freedom of both movement and space(Anderson-Hanley et al., 2003) In light of this, it is therefore important that residential care should consider offering choices and supporting elderly people from BSSA communities to be able to fulfil their preferred outdoor life with people familiar to them. This can be a good way of ameliorating loneliness and boredom through promoting outdoor life and wider community interaction. Many elderly people across the social divide are affected by loneliness, which can lead to other physical health problems (Department of Health, 2014). Though this may attract costs that may increase the financial burden on the elderly people, there is need to consider new interventions to combat loneliness and restricted movement in residential care. This can benefit many elderly people not only from the BSSA communities but also from the wider population. The notion of confinement is totally opposed to the strongly held beliefs of "Ubuntu” among BSSA communities (Letseka, 2011). Ubuntu when translated from different Bantu languages means 'I am because we are' or a 'person is a person through other people.' This concept gives rise to the perception that elderly people among BSSA communities should continue to be part of the community until death as any separation to live outside the community may be viewed as rejection.

One of the lasting legacy among BSSA communities is to live around and show your material and physical achievements to the young generation and the community at large (Frobenius, 2007). Ownership of material gains ranging from immovable property to movable is used as a yardstick for success in life. The research participants felt that moving into residential care diminishes their legacy and ability to own materials and property accumulated during their lifetime. This in turn takes away their pride and confidence, which is usually associated with success achieved by individuals during their lifetime sometimes leading to depression and anxiety. It is therefore important that residential care takes into cognisance such cultural values and find some creative ways in which they can be recognised and valued. It is also 
equally important that new communities are educated and appraised on the value of residential care including its recognition and respect for individuals' cultural values and beliefs.

Different groups of people have relationships with food and reveals an abundance of information about their history and identity. A person 's beliefs, passions, background knowledge, assumptions and personalities can be linked to food. Hauck-Lawson (2004) coined the concept of food voice. In the conceptual framework, she suggested the notion that what one eats or chooses not to eat communicates aspects of a person's identity or emotion in a manner that words alone cannot describe (Hauck-Lawson, 2004). The BSSA research participants attached so much importance on the nature and type of food available in residential care. They believed that the type of food served in residential care did not include their traditional dishes and were therefore sceptical about taking up residential care in their later years. Considering this assertion, there is need for residential care providers to consider providing different food catering for diverse populations being served. Such a shift can also redefine the concept of diversity and inclusivity in the British residential care system. Thus, opening up to new communities.

African culture is embedded in strong moral considerations and beliefs (Idang, 2015). It consists of a system of various beliefs and customs which every individual ought to keep as part of their values and identity. It is in this notion that personal care on the elderly is supposed to be performed by close friends or relatives. This signifies the concept of "Ubuntu" (humanity) at play where the young people ought to fulfil their obligation of supporting the custodians of their communities (Ezedike, 2009). The BSSA research participants were concerned of being given personal care by strangers and at times not in the way they do it when they take up residential care. Again, considering this, it is important that residential care providers should consider training their staff in cultural competency to include personal care and desist from providing a generic personal care approach to a diverse of individuals (Ali, 1997). Such considerations can also include educational awareness within the BSSA communities on how the care providers recognises diversity of the communities they serve.

Lanre-Abass (2008) asserts that, African societies are communalistic and do not acknowledge advance care directives which according to many Africans encourage "atomistic individualism" (Lanre-Abass, 2008). Atomistic individualism in this case refers to the idea 
that the isolated individual is the only fundamental reality and that the individual is the natural atom in artificial social composite (Umoh, 2012). In Africa, people grow up with a sense of belonging and relatedness with others (Sanchez-Conzalez, 1997). Individuals, thus, have a sense of obligation to a larger set of other individuals. Following this notion, many BSSA communities do not subscribe to advance care directives and such decisions are left to close relatives who normally perform palliative care on the individual concerned. This notion supports the reason given by BSSA research participants that if they take up residential care they are likely going to die in the care of strangers as opposed to their cultural beliefs. In light of this, it is therefore imperative that residential care providers increase the level of relatives' involvement to cater for cultural and social needs of such communities during end of life care.

Many African communities believe that during the time of difficulties, those who are well should take care of the weak and frail as a demonstration of "Ubuntu" - (humanity) (Mbiti, 1990). From this context, taking a relative to a residential care institution is normally viewed as being neglected by relatives and is highly stigmatised within the African communities. Considering the changing social and physical environment following migration to Europe, it is important to raise awareness among BSSA communities on the rationale and importance of residential care. This will reduce the stigma and increase uptake of residential care.

Exclusion of individuals living in residential care can lead to loneliness, resentment and subsequent stress (Psychiatrists, 2009). Pass time activities are closely related to culture and identity of individuals (Ali, 1997). BSSA research participants felt that some of the activities that are done in residential care did not resonate with their culture and may therefore feel excluded leading to loneliness and stress (Thornicroft, 2006). Considering the diversity of individuals coming into residential care, it is important for care providers to widen the scope of their engagement activities to cater for a wide variety of population from different cultural backgrounds. More importantly as alluded earlier on, it is also imperative that staff working in residential care are trained in cultural competency in order for them to be able to work with a diverse population.

\section{Implications for practice}

There is need to understand the needs and concerns of new communities in relation to residential care and make necessary changes to enhance diversity and inclusivity. More importantly, the curriculum and professional development courses for staff in health and 
social care need to factor in the concepts of cultural competency and inclusivity to prepare them for the increasingly changing terrain of social care. Furthermore, new communities need to be made aware of the already existing residential care services and invite them for contributions in designing a non-generic residential care service which reflects different cultural dimensions.

\section{Limitations of the study}

Future research needs to utilise a mixed-method approach to deal with a wide variety of issues and enhance generalisability of the issues under consideration. There is need for crosscultural comparisons of BSSA communities living in the UK and BSSA communities living in Africa or other parts of the world. This may enhance understanding the differences and similarities based on contextual social, political and economic factors.

\section{Concluding comments}

Owing to the changing demography and diversity in the UK population, there is need to reorient and redesign residential care services provision to make it diverse and inclusive of new communities from other cultures (Calvert 2020). It is also important that awareness to increase the uptake of residential care among new communities like the BSSA communities should be considered in collaboration with the health promotion sectors. Training of residential care staff should be made a priority and promote cultural competency and enhance inclusivity when serving diverse communities (Schouler-Ocak et al 2015). More research may be needed to understand and improve the experiences of new social groups like BSSA communities in residential care.

\section{References}

Ali, S. (1997). A Guide to Anti-racism Organizational Change in Health and Mental Health Sector: Across Boundaries.

ANDERSON-HANLEY, C., MESHBERG, S. R. and MARSH, M. A. (2003). The effects of a control-enhancing intervention for nursing home residents: Cognition and locus of control as moderators. Palliative \& supportive care, 1(2), 111-120.

Calvert, P. (2020). Submission to the Royal Commission Into Aged Care Quality and Safety: Aged Care Program Redesign.

Centre for Ageing Better (2017). Work and health in later life, Centre for Ageing Better.: Retrieved from https://www.ageing-better.org.uk/news/work-and-health-later-life Department of Health (2009). Black and Minority Ethnic (BME): Positive Practice Guide, 
Improving Access to Psychological Therapies.

Dyall, L., Kepa, M., Hayman, K., Teh, R., Moyes, S., Broad, J. B. and Kerse, N. (2013). Engagement and recruitment of Māori and non-Māori people of advanced age to LiLACS NZ. Australian and New Zealand Journal of Public Health, 37(2), 124-131.

Ezedike, E. (2009). African Culture and the African Personality: From Footmarks to Landmarks on African Philosophy: Somolu. Obaroh and Unwin.

Frobenius, L. (2007). Leo Frobenius on African History, Art and Culture: An Anthology: Markus Wiener Pub.

Hauck-Lawson, A. (2004). Introduction to special issue on the food voice. Food, Culture, and Society, 7(1), 24-25.

Holland, C. A., Everitt, P., Johnson, A. and Devi, R. (2008). The 'Healthy Passport'intervention with older people in an English urban environment: effects of incentives and peer-group organisers in promoting healthy living. Ageing \& Society, 28(4), 525-549.

Idang, G. E. (2015). African culture and values. Phronimon, 16(2), 97-111.

Ifekwunigwe, J. O. (1997). Diaspora's daughters, Africa's orphans? Black British feminism: A reader, 127.

Lanre-Abass, B. A. (2008). Cultural Differences in Advance Directives Relating to End of Life Decision Making. Prajñā Vihāra, 9(1).

Letseka ,M.(2011 ) Educating for Ubuntu: Open Journal of Philosophy[online]available at ww.scirp.org/journal/PaperDownload.aspx?paper ID=32164 [accessed:16 March 2018]

Martinez, I. L., Kim, K., Tanner, E., Fried, L. P., \& Seeman, T. (2009). Ethnic and class variations in promoting social activities among older adults. Activities, adaptation \& aging, 33(2), 96-119.

Maybin, J., Charles, A., \& Honeyman, M. (2016). Understanding quality in district nursing: learning from the experiences of patients, carers and staff: London: The King's Fund. Available at: www. kingsfund. org. uk/publications ....

Office of National Statistics, UK (2014) Health \& Social Care https://www.ons.gov.uk/peoplepopulationandcommunity

Patton, M.Q. (2002). Two decades of developments in qualitative inquiry: A personal, experiential perspective. Qualitative social work, 1(3), pp.261-283. 
Psychiatrists, R. (2009). Mental Health and Social Inclusion: Making Psychiatry and Mental Health Services Fit for the 21st Century. Royal College of Psychiatrists.

Sanchez-Conzalez, M. A. (1997). Advance directives outside the USA: are they the best solution everywhere? Theoretical medicine, 18, 283-301.

Schouler-Ocak, M., Graef-Calliess, I. T., Tarricone, I., Qureshi, A., Kastrup, M. C., \& Bhugra, D. (2015). EPA guidance on cultural competence training. European Psychiatry, 30(3), 431-440.

Serrant-Green, L. (2011). The sound of 'silence': a framework for researching sensitive issues or marginalised perspectives in health. Journal of Research in Nursing, 16(4), 347-360.

The Centre for Mental Health. (2002). Breaking the circles of fear. A review of the relationship between mental health services and African and Caribbean communities. London: The Sainsbury Centre for Mental Health, (Vol. ISBN: 1 $870480554)$.

Thornicroft, G. (2006). Shunned: Discrimination against people with mental illness (Vol. 399): Oxford university press Oxford.

Torres, S. (2012). Healthy aging among immigrants in Sweden: what we know and need to find out Healthy Aging in Sociocultural Context (pp. 60-68): Routledge.

Umoh, D. S. (2012). Death is not natural: The African story. 